\title{
A simple asynchronous distributed STBC network scheme with full diversity
}

\author{
Wei Song ${ }^{1 *}$, Qiang Liư ${ }^{2}$ and Moon Ho Lee ${ }^{3}$
}

\begin{abstract}
In this article, we propose a simple distributed space-time block code (STBC) network scheme for asynchronous relay network, where every two traditional relay nodes are combined to one node to amplify and forward the processed signals via the same transmission antenna. In the proposed distributed network scheme, we consider a wireless scenario with frequency selective fading channels. The orthogonal frequency division multiplexing (OFDM) symbols are broadcast from source to relay nodes in the first step, and in the second step, the received signals at the relay nodes are implemented with a simple operation and mixed partially and forwarded to destination. In this network scheme, the four by four quasi-orthogonal STBC (QO-STBC) is applied, and the orthogonal decoding algorithm can be used at the receiver because of the orthogonality of the re-constructed equivalent transmission matrix. Thus, the full rate and the full diversity are achieved at the transmitter and the receiver of the proposed distributed network scheme, respectively. Simulation result shows that the proposed distributed STBC network scheme has better performance than other network schemes.
\end{abstract}

Keywords: OFDM, Distributed network, Space-time code, Quasi-orthogonal, Full diversity

\section{Introduction}

The space-time block codes (STBC) can provide an effective capacity gain and spatial diversity gain in multipleinput and multiple-output systems. It is well known that a "good" STBC is an orthogonal STBC (O-STBC) with the full rate and the full diversity, and it has linear decoding complexity. The first full rate O-STBC scheme was proposed by Alamouti [1] for two transmission antennas. Subsequently, O-STBC design theory was issued by Tarokh et al. [2]. The authors proved that the full rate and the full diversity complex O-STBCs did not exist for more than two transmission antennas. Consequently, Jafarkhani constructed a quasi-orthogonal STBC (QO$\mathrm{STBC}$ ) design [3] with the full rate. But the diversity gain of the QO-STBC was reduced, and the decoding complexity was increased linearly. The QO-STBC with pairwise decoding has better performance than the codes with rate $<1$ from orthogonal design at low signalto-noise ratio (SNR), and worse at high SNR due to diversity loss. Tirkkonen, Boariu, and Hottinen (TBH)

\footnotetext{
*Correspondence: sw658@hotmail.com

${ }^{1}$ College of Information Technology, Eastern liaoning university, Dandong, 118003, P. R. China

Full list of author information is available at the end of the article
}

also proposed a scheme [4], and their behaviors are similar to the Jafarkhani's QO-STBC. These QO-STBC designs can get the full rate codes, but the codes can not achieve the full diversity. The interference terms comes from neighboring signals and it leads to the increase of the decoding complexity and the decrease of the performance. To achieve full diversity for quasi-orthogonal codes, constellation rotation approaches were proposed in [5] and the authors gave optimal rotation angles for PSK and QAM constellations. Anyway, orthogonal spacetime codes have played important roles in communication system due to good performance and low decoding complexity.

With the relay scheme considered, many articles become more concerned with the cooperative relay scheme. In [6,7], a variety of low complexity cooperative diversity protocols and an alternative approach based on space time codes, which allows all partners to transmitter on the same sub-channel, are proposed. Full diversity without any feedback can be achieved through space time coded cooperative diversity. Also, the potential benefits of cooperation are indicated by the cooperation information theoretic capacity, outage, and coverage analysis in $[8,9]$.

\section{每 Springer}


Recently, the distributed STBC network schemes have been given significant amount of attention. In [10], the authors used two-stage protocol to apply distributed space-time coding where the special cases, which the Alamouti code and the Jafarkhani code, were included. The paper shows that the coding gain and the diversity gain were the same as the equivalent multiple-antenna (MA) system. To improve effective transmission rate, the orthogonal frequency division multiplexing (OFDM) symbols were applied in distributed network scheme [11], the authors implemented the Alamouti OFDM code in two way relay network, and the full rate and the full diversity were achieved. However, the cooperative relay schemes always use two stages, the first of which is from source to relays, and the second step is from relays to destination. So, the total time slots is double of MA and the rate is half of MA. Then, the full rate is more important in multi-hop network. And the Alamouti code is always used in network as the rate O-STBC block, which means that the maximum diversity gain cannot be more than 2.

In this article, we propose a new distributed STBC network scheme with two relay nodes, in which each relay node is equipped with two receiving antennas and one transmission antenna and the equivalent Q-STBC is implemented. The proposed distributed network scheme achieves the full rate and the full diversity at the process of transmitter and receiver, respectively, and the maximum diversity gain of the transmission code block can be increased. In the following sections, $A^{T}$ denotes the transpose, $A^{*}$ denotes the conjugate, and $A^{H}$ denotes the conjugate transpose.

This article is outlined as follows. In Section "System model", we describe the distributed network scheme model. In Section "A simple distributed STBC network scheme", we propose the simple distributed STBC network scheme and analyze its performance. Section "Simulation results", simulation results are presented by comparing the proposed distributed network scheme with the previous schemes. Finally, conclusions are drawn in the Section "Conclusions".

\section{System model}

Considering a relay network with one source node $S$, two relay nodes $R_{1}$ and $R_{2}$, and one destination node $\mathrm{D}$ over a wireless multi-hop channel, where each relay node has one transmission antenna and two receiving antennas, and the source node and the destination node have a single antenna respectively, all nodes working in half duplex mode, as shown in Figure 1. We assume that the communication system is performed over frequency selective fading channels, in which the distance from the source node to the destination node is so far that the source node can not communicate with the destination node directly.

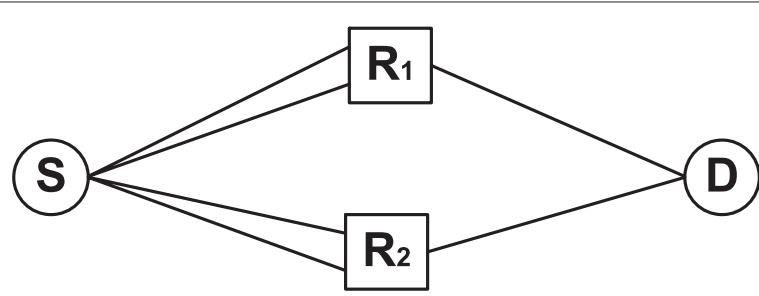

Figure 1 The block diagram of the proposed DSTBC network scheme.

In this proposed scheme, each relay node can be seen as one block which is made up of two traditional relay with single-antenna using the same transmission antenna simply.

The $S \rightarrow R_{i}$ channels are assumed to be a frequency selective channel with $\mathrm{L}$ independent propagation paths. Besides, we assume that the channel state information is quasi-static, so we can write the channel impulse response as

$$
h_{S, R_{i}^{j}}(t)=\sum_{l=0}^{L-1} \alpha_{S, R_{i}^{j}}(l) \delta\left(t-\tau_{l, i}\right)
$$

where $\alpha_{S, R_{i}^{j}}$ is the channel coefficient of the $l$ th path form the source $\mathrm{S}$ to the $j$ th antenna of $R_{i}$, and $\tau_{l, i}$ denotes the corresponding path delay.

First, at the source node $S$, the information bits are modulated and mapped into $M$ OFDM symbols with $N$ subcarriers by $N$-point FFT as $X_{k}=\left[x_{k, 0}, x_{k, 1}, \ldots, x_{k, N-1} \mid\right.$ $k \in M]$. Then each OFDM symbol is preceded by the a cyclic prefix $(\mathrm{CP})$ with length $l_{S R}$. For simplicity, we select four OFDM symbols as a block to broadcast from the source $\mathrm{S}$ to the destination $\mathrm{D}$ via the relay nodes $R_{i}$. For the proposed system model, there are suppositions:

- The destination node D knows all the channel state information $h_{S, R_{i}^{j}}(l)$ and $h_{R_{i}, D}(l)$.

- The channel state information $h_{S, R_{i}^{j}}(l)$ and $h_{R_{i}, D}(l)$ are independent and identically-distributed.

- All nodes including $S, D$, and $R_{i}$, have been subjected to half duplex mode.

\section{A simple distributed STBC network scheme}

In this section, we propose a simple distributed STBC network scheme with time-removal processing. Considering two kind of timing errors [11], in this proposed scheme, without loss of generality, we assume that the signals arrived at the destination $D$ via the relay node $R_{2}$ earlier. In fact, in this proposed scheme, the second kind of timing errors are more harmful than the first kind of timing error, because the real mixed signals happen at the destination node. So, $l_{S, R}$ should be chosen to keep the orthogonality of OFDM symbol suitably. At the second 
hop, we assume that the OFDM symbols via the relay node $R_{2}$ arrived at the destination $\mathrm{D}$ with $\tau_{R_{2}, S}$ samples faster. In order to combat the second kind of timing errors, the OFDM symbols remove the $\mathrm{CP}$ with length $l_{S, R}$ at the relay node $R_{2}$, first and then add the CP with length $l_{R, S}$, where $l_{R, S}>\max \left\{l_{S, R}, \tau_{R_{2}, S}\right\}$.

Furthermore, according to optimal power allocation [10], assuming that $P$ is the total transmission power in the whole scheme. Thus, the optimum power allocation is that the source node uses half of the total power and relay nodes share the other half, i.e., $P_{1}=2 P_{2}=P / 2$, where $P_{1}$ and $P_{2}$ are the average power at the source node $S$ and the relay node $R_{i}$, respectively. Following the upper description, at each antenna of the relay nodes $R_{i}$ of the $k$ th time slot, we have the received signals as

$$
r_{S, R_{i}^{j}}^{k}=\sqrt{P_{1}}\left(F F T\left(X_{k}\right) \circledast h_{S, R_{i}^{j}}\right)+n_{i, j}^{k},
$$

where $\circledast$ denotes the circular convolution, and $n_{i, j}^{k}$ is the additive white Gaussian noise (AWGN) with zero-mean and unit-variance from the source $S$ to the antenna $R_{i}^{j}$ at the $k$ th time slot. After four OFDM symbols arrived, the received signals can be processed as Table 1 .

And two received OFDM signals of each relay node are combined and forwarded to destination $\mathrm{D}$ with a scalar $\rho=\sqrt{\frac{P_{2}}{2 P_{1}+1}}$ during four time slots. Note that, at the relay $R_{2}$, the CP should be updated before the mixed signals are transmitted. The proceed mixed signals as Table 2, where $\zeta($.) represents the time-removal operation.

At the destination node $D$, after $\mathrm{CP}$ removal, the received signals for four successive OFDM symbols duration can be written as

$$
\begin{aligned}
z_{1}= & \sqrt{\frac{P_{2}}{2 P_{1}+1}}\left(\left(r_{S, R_{1}^{1}}^{1}+r_{S, R_{1}^{2}}^{2}\right) \circledast h_{R_{1}, D}\right. \\
& \left.+\zeta\left(r_{S, R_{2}^{1}}^{3}+r_{S, R_{2}^{2}}^{4}\right) \circledast h_{R_{2}, D}\right)+w_{1},
\end{aligned}
$$

$$
\begin{aligned}
z_{2}= & \sqrt{\frac{P_{2}}{2 P_{1}+1}}\left(\left(\left(r_{S, R_{1}^{2}}^{1}\right)^{*}-\left(r_{S, R_{1}^{1}}^{2}\right)^{*}\right) \circledast h_{R_{1}, D}\right. \\
& \left.+\zeta\left(\left(r_{S, R_{2}^{2}}^{3}\right)^{*}-\left(r_{S, R_{2}^{1}}^{4}\right)^{*}\right) \circledast h_{R_{2}, D}\right)+w_{2}, \\
z_{3}= & \sqrt{\frac{P_{2}}{2 P_{1}+1}}\left(\left(r_{S, R_{1}^{1}}^{3}+r_{S, R_{1}^{2}}^{4}\right) \circledast h_{R_{1}, D}\right. \\
& \left.+\zeta\left(r_{S, R_{2}^{1}}^{1}+r_{S, R_{2}^{2}}^{2}\right) \circledast h_{R_{2}, D}\right)+w_{3}, \\
z_{4}= & \sqrt{\frac{P_{2}}{2 P_{1}+1}}\left(\left(\left(r_{S, R_{1}^{2}}^{3}\right)^{*}-\left(r_{S, R_{1}^{1}}^{4}\right)^{*}\right) \circledast h_{R_{1}, D}\right. \\
& \left.+\zeta\left(\left(r_{S, R_{2}^{2}}^{1}\right)^{*}-\left(r_{S, R_{2}^{1}}^{2}\right)^{*}\right) \circledast h_{R_{2}, D}\right)+w_{4},
\end{aligned}
$$

where $w_{i}(i=1,2,3,4)$ is also the AWGN with zero-mean and unit-variance from the relay node to the destination

\begin{tabular}{|c|c|c|c|}
\hline $\begin{array}{l}r_{S, R_{1}^{1}}^{1} \\
r_{S, R_{1}^{2}}^{2} \\
r_{S, R_{2}^{1}}^{3} \\
r_{S, R_{2}^{2}}^{4}\end{array}$ & $\begin{array}{c}\left.-\left(r_{S, R}^{2}\right)_{1}\right)^{*} \\
\left(r_{S, R_{1}^{2}}^{1}\right)^{*} \\
-\left(r_{S, R_{2}}^{4}\right)^{*} \\
\left(r_{S, R_{2}^{2}}^{3}\right)^{*}\end{array}$ & $\begin{array}{l}r_{S, R_{1}^{1}}^{3} \\
r_{S, R_{1}^{2}}^{4} \\
r_{S, R_{2}^{1}}^{1} \\
r_{S, R_{2}^{2}}^{2}\end{array}$ & $\begin{array}{c}-\left(r_{S, R_{1}^{1}}^{4}\right)^{*} \\
\left(r_{S, R_{1}^{2}}^{3}\right)^{*} \\
-\left(r_{S, R_{2}^{1}}^{2}\right)^{*} \\
\left(r_{S, R_{2}^{2}}^{1}\right)^{*}\end{array}$ \\
\hline
\end{tabular}
node $D$ at the $i$ th time slot.

Then, the received signals at $D$ are fed to $N$-point FFT and IFFT operation, respectively, since the length of CP is long enough. Following the property $\operatorname{FFT}\left(\zeta\left(F F T\left(X_{k}\right)\right)\right)=$ $\operatorname{IFFT}\left(F F T\left(X_{k}\right)\right)$ [12], we can get the separating received signals as follows:

$$
\begin{aligned}
& Z_{1}^{1}=\rho \operatorname{IFFT}\left(\left(r_{S, R_{1}^{1}}^{1}+r_{S, R_{1}^{2}}^{2}\right) \circledast h_{R_{1}, D}\right)+\operatorname{IFFT}\left(w_{1}^{1}\right), \\
& Z_{1}^{2}=\rho F F T\left(\zeta\left(r_{S, R_{2}^{1}}^{3}+r_{S, R_{2}^{2}}^{4}\right) \circledast h_{R_{2}, D}\right)+F F T\left(w_{1}^{2}\right), \\
& Z_{2}^{1}=\rho \operatorname{IFFT}\left(\left(\left(r_{S, R_{1}^{2}}^{1}\right)^{*}-\left(r_{S, R_{1}^{1}}^{2}\right)^{*}\right) \circledast h_{R_{1}, D}\right)+\operatorname{IFFT}\left(w_{2}^{1}\right), \\
& Z_{2}^{2}=\rho F F T\left(\zeta\left(\left(r_{S, R_{2}^{2}}^{3}\right)^{*}-\left(r_{S, R_{2}^{1}}^{4}\right)^{*}\right) \circledast h_{R_{2}, D}\right)+F F T\left(w_{2}^{2}\right), \\
& Z_{3}^{1}=\rho \operatorname{IFFT}\left(\left(r_{S, R_{1}^{1}}^{3}+r_{S, R_{1}^{2}}^{4}\right) \circledast h_{R_{1}, D}\right)+\operatorname{IFFT}\left(w_{3}^{1}\right), \\
& Z_{3}^{2}=\rho F F T\left(\zeta\left(r_{S, R_{2}^{1}}^{1}+r_{S, R_{2}^{2}}^{2}\right) \circledast h_{R_{2}, D}\right)+F F T\left(w_{3}^{2}\right),
\end{aligned}
$$

Table 1 The received signals

Table 2 The proceed mixed signals

\begin{tabular}{lcc}
\hline & \multicolumn{1}{c}{$\boldsymbol{R}_{\mathbf{1}}$} & $\boldsymbol{R}_{\mathbf{2}}$ \\
\hline 1st-slot & $r_{S, R_{1}^{1}}^{1}+r_{S, R_{1}^{2}}^{2}$ & $\zeta\left(r_{S, R_{2}^{1}}^{3}\right)+\zeta\left(r_{S, R_{2}^{2}}^{4}\right)$ \\
2nd-slot & $\left(r_{S, R_{1}^{1}}^{1}\right)^{*}-\left(r_{S, R_{1}^{2}}^{2}\right)^{*}$ & $\zeta\left(\left(r_{S, R_{2}^{3}}^{3}\right)^{*}\right)-\zeta\left(\left(r_{S, R_{2}^{1}}^{4}\right)^{*}\right)$ \\
3rd-slot & $r_{S, R_{1}^{1}}^{3}+r_{S, R_{1}^{2}}^{4}$ & $\zeta\left(r_{S, R_{2}^{1}}^{1}\right)+\zeta\left(r_{S, R_{2}^{2}}^{2}\right)$ \\
4th-slot & $\left(r_{S, R_{1}^{3}}^{3}\right)^{*}-\left(r_{S, R_{1}}^{4}\right)^{*}$ & $\zeta\left(\left(r_{S, R_{2}^{1}}^{1}\right)^{*}\right)-\zeta\left(\left(r_{S, R_{2}^{1}}^{2}\right)^{*}\right)$ \\
\hline
\end{tabular}




$$
\begin{aligned}
& \left.Z_{4}^{1}=\rho \operatorname{IFFT}\left(\left(r_{S, R_{1}^{2}}^{3}\right)^{*} !-\left(r_{S, R_{1}^{1}}^{4}\right)^{*}\right) \circledast h_{R_{1}, D}\right)+\operatorname{IFFT}\left(w_{4}^{1}\right), \\
& Z_{4}^{2}=\rho F F T\left(\zeta\left(\left(r_{S, R_{2}^{2}}^{1}\right)^{*}-\left(r_{S, R_{2}^{1}}^{2}\right)^{*}\right) \circledast h_{R_{2}, D}\right)+F F T\left(w_{4}^{2}\right),
\end{aligned}
$$

where $w_{k}^{j}(j=1,2)$ is the component of $w_{k}$.

After getting the separated signals, the scheme performs the addition and subtraction operations between two correlative separated signals $Z_{k}^{1}$ and $Z_{k}^{2}$, respectively. The two new received signals are denoted as

$$
\begin{aligned}
& Y_{k, 1}=Z_{k}^{1}+Z_{k}^{2}, \\
& Y_{k, 2}=Z_{k}^{1}-Z_{k}^{2},
\end{aligned}
$$

It means that two new received signals are obtained at each time interval. Finally, we can get an

$$
\mathbf{Y}=\beta \mathbf{S H}+\mathbf{n},
$$

where the scalar $\beta=\sqrt{\frac{P_{1} P_{2}}{2 P_{1}+1}}, \mathbf{Y}$ is a $8 \times 1$ vector consisted of received signals, and

$$
\mathbf{H}=\left[h_{S, R_{1}^{1}} h_{R_{1}, D} h_{S, R_{1}^{2}} h_{R_{1}, D} h_{S, R_{2}^{1}} h_{R_{2}, D} h_{S, R_{2}^{2}} h_{R_{2}, D}\right]^{T}
$$

and $\mathbf{S}$ and $\mathbf{n}$ are the equivalent $8 \times 4$ transmission matrix and $8 \times 1$ noise vector, respectively.

We next analyze the orthogonality of the transmission matrix $\mathbf{S}$. We have

$$
\mathbf{S}=\left[\begin{array}{ccccccccc}
X_{1} & -X_{2}{ }^{*} & -X_{3}{ }^{*} & X_{4} & X_{1} & -X_{2}{ }^{*} & -X_{3}{ }^{*} & X_{4} \\
X_{2} & X_{1}{ }^{*} & -X_{4}{ }^{*} & -X_{3} & X_{2} & X_{1}{ }^{*} & -X_{4}{ }^{*} & -X_{3} \\
X_{3} & -X_{4}{ }^{*} & X_{1}{ }^{*} & -X_{2} & -X_{3} & X_{4}{ }^{*} & -X_{1}^{*} & X_{2} \\
X_{4} & X_{3}{ }^{*} & X_{2}{ }^{*} & X_{1} & -X_{4} & -X_{3}{ }^{*} & -X_{2}{ }^{*} & -X_{1}
\end{array}\right]^{T}
$$

Obviously, we can rewrite $\mathbf{S}=\left[\begin{array}{ll}Q_{J} & \widehat{Q}_{J}\end{array}\right]^{T}$, where $Q_{J}$ is the Jafarkhani's transmission matrix [3], and $\widehat{Q}_{J}$ is the transformation matrix of $Q_{J}$, and $\widehat{Q}_{J}=Q_{J} \Lambda$, where $\Lambda=$ $\operatorname{diag}[1,1,-1,-1]$ is a diagonal matrix. Also, we have

$$
Q_{J}^{H} Q_{J}=\left[\begin{array}{cccc}
\alpha & 0 & 0 & \beta_{J} \\
0 & \alpha & -\beta_{J} & 0 \\
0 & -\beta_{J} & \alpha & 0 \\
\beta_{J} & 0 & 0 & \alpha
\end{array}\right],
$$

and

$$
\widehat{Q}_{J}^{H} \widehat{Q}_{J}=\Lambda Q_{J}^{H} Q_{J} \Lambda=\left[\begin{array}{cccc}
\alpha & 0 & 0 & -\beta_{J} \\
0 & \alpha & \beta_{J} & 0 \\
0 & \beta_{J} & \alpha & 0 \\
-\beta_{J} & 0 & 0 & \alpha
\end{array}\right],
$$

where $\alpha=\sum_{i=1}^{4}\left|X_{i}\right|^{2}$ and $\beta_{J}=\left(X_{1} X_{4}^{*}+X_{1}^{*} X_{4}\right)-\left(X_{2} X_{3}^{*}+\right.$ $\left.X_{2}^{*} X_{3}\right)$.

So the character matrix of $\mathbf{S}$ can be written as

$$
\mathbf{S}^{H} \mathbf{S}=\left[\begin{array}{cccc}
2 \alpha & 0 & 0 & 0 \\
0 & 2 \alpha & 0 & 0 \\
0 & 0 & 2 \alpha & 0 \\
0 & 0 & 0 & 2 \alpha
\end{array}\right]
$$

From (13), we know that the equivalent transmission code $\mathbf{S}$ is a new kind of half rate O-STBC, it can achieve full diversity four. And comparing with the original Jafarkhani code, the coding gain is also increased.

In this proposed scheme, we can apply the Jafarkhani code to achieve full rate code at the transmitter, and full diversity at the receiver. The fast linear decoding algorithm [2] can be used, where we can omit the superscript of matrix elements for simplicity, as following.

Let $\epsilon_{t}$ denotes the permutations of the symbols from the first row to the $t$ th row of the transmission matrix. The column position of $X_{i}$ in the $t$ th row is represented by $\epsilon_{t} \quad(i)$ and the sign of $X_{i}$ in the $t$ th row is denoted by $\operatorname{sgn}_{t}(i)$. Based on the orthogonality of the equivalent orthogonal code matrix $\mathbf{S}$, the decision statistics $\widetilde{X}_{i}$ of the transmitted signal $X_{i}$ can be constructed as

$$
\widetilde{X}_{i}=\sum_{t \in \eta(i)} \operatorname{sgn}_{t}(i) \cdot \tilde{y}_{t} \cdot \tilde{h}_{\in_{t}(i)}^{*},
$$

where $\eta(i)$ is the set of rows of the transmission matrix including $x_{i}$,

$$
\begin{aligned}
& \tilde{y}_{t}(i)=\left\{\begin{array}{l}
y_{t}, \quad X_{i} \text { belongs to the } t \text { th row of } \mathbf{S} ; \\
\left(y_{t}\right)^{*}, X_{i}^{*} \text { belongs to the } t \text { th row of } \mathbf{S},
\end{array}\right. \\
& \tilde{h}_{\in_{t}(i)}^{*}=\left\{\begin{array}{l}
h_{\epsilon_{t}(i)}^{*}, X_{i} \text { belongs to the } t \text { th row of } \mathbf{S} ; \\
h_{\in_{t}(i)}, X_{i}^{*} \text { belongs to the } t \text { th row of } \mathbf{S} .
\end{array}\right.
\end{aligned}
$$

Since the value of $\tilde{X}_{i}$ only depends on the code symbol $X_{i}$, the given received signals, the path coefficients and the structure of the transmission matrix are minimizing each individual decision metric as

$$
\left|\tilde{X}_{i}-X_{i}\right|^{2}+\left(2 \sum_{j=1}^{4}\left|h_{j}\right|^{2}-1\right)\left|X_{i}\right|^{2}
$$


Applying (14), we obtain the following decision statistics:

$$
\begin{aligned}
\tilde{X}_{1}= & \gamma X_{1}+a_{1}\left(h_{S, R_{1}} h_{R_{1}, D}\right)^{*}+a_{2}\left(h_{S, R_{1}^{2}} h_{R_{1}, D}\right) \\
& +a_{3}\left(h_{S, R_{2}^{1}} h_{R_{2}, D}\right)+a_{4}\left(h_{S, R_{2}^{2}} h_{R_{2}, D}\right)^{*}, \\
\tilde{X}_{2}= & \gamma X_{2}-a_{2}\left(h_{S, R_{1}^{1}} h_{R_{1}, D}\right)+a_{1}\left(h_{S, R_{1}^{2}} h_{R_{1}, D}\right)^{*} \\
& -a_{4}\left(h_{S, R_{2}^{1}} h_{R_{2}, D}\right)^{*}+a_{3}\left(h_{S, R_{2}^{2}} h_{R_{2}, D}\right), \\
\widetilde{X}_{3}= & \gamma X_{3}+a_{8}\left(h_{S, R_{1}^{1}} h_{R_{1}, D}\right)-a_{7}\left(h_{S, R_{1}^{2}} h_{R_{1}, D}\right)^{*} \\
& +a_{5}\left(h_{S, R_{2}^{1}} h_{R_{2}, D}\right)^{*}-a_{6}\left(h_{S, R_{2}^{2}} h_{R_{2}, D}\right), \\
\tilde{X}_{4}= & \gamma X_{4}+a_{7}\left(h_{S, R_{1}^{1}} h_{R_{1}, D}\right)^{*}+a_{8}\left(h_{S, R_{1}^{2}} h_{R_{1}, D}\right) \\
& +a_{6}\left(h_{S, R_{2}^{1}} h_{R_{2}, D}\right)+a_{5}\left(h_{S, R_{2}^{2}} h_{R_{2}, D}\right)^{*},
\end{aligned}
$$

where

$$
\begin{array}{ll}
a_{1}=n_{1}+n_{5}, & a_{2}=n_{2}^{*}+n_{6}^{*}, \\
a_{3}=n_{3}^{*}-n_{7}^{*}, & a_{4}=n_{4}-n_{8}, \\
a_{5}=n_{1}-n_{5}, & a_{6}=-n_{2}^{*}+n_{6}^{*}, \\
a_{7}=n_{4}+n_{8}, & a_{8}=-n_{3}^{*}-n_{7}^{*},
\end{array}
$$

and $\gamma=2 \sum_{i=1}^{2} \sum_{j=1}^{2}\left|h_{S, R_{i}^{j}} h_{R_{i}, D}\right|^{2} . n_{i}$ denotes the $i$ th element of the noise vector $\mathbf{n}$.

The whole scheme can achieve the full rate and the full diversity at the process of transmission and the receiver, respectively. Evidently the decoding complexity is reduced and the performance is improved, though the complexity of the receiver is increased. Also, we know that the proposed distributed network scheme has a higher coding gain than conventional QO-STBC. Furthermore, this scheme can be achieved by using the TBH code.

\section{Simulation results}

In this section, we show the simulation performance of the proposed distributed STBC network scheme for the radiation-power-limited communication system. In our simulation, we considered the assumption of frequency selective Rayleigh flat fading channels by employing the ML-based decoding algorithm, and we select only one block-code to compare the performance with conventional distributed STBCs.

In this article, it is obvious that the rate of the system equals to the half of the rate of the multiple-antenna system (RMA) due to the two-hop channel model selected. Figure 2 shows the simulations, in which we choose the proposed distributed STBC network scheme with RMA 1 , the conventional distributed QO-STBC scheme $[3,10]$ with RMA 1, the distributed O-STBC network scheme [2] with RMA $\frac{1}{2}$ and the Alamouti-base OFDM network scheme [11] with RMA 1 to compare the performance. As we known, the diversity order of the Alamouti-base OFDM network scheme is two, for the sake of fairness, we select two-block layered Alamouti network scheme, the transmission matrix can be written as

$$
\mathbf{S}_{L}=\left[\begin{array}{cccc}
x_{1} & -x_{2}^{*} & x_{3} & -x_{4}^{*} \\
x_{2} & x_{1}^{*} & x_{4} & x_{3}^{*}
\end{array}\right]^{T}
$$

So the RMA of the layered Alamouti scheme comes to 2. And for each block-code, the diversity gain of the proposed distributed STBC network scheme, the conventional distributed QO-STBC scheme, the distributed O-STBC network scheme and the Alamouti-base OFDM network schemes are $4,<4,4$, and 2 , respectively.

Figure 2a shows the simulations, in which we compared the performance of our proposed distributed STBC network scheme (QPSK) with the layered Alamouti network scheme (BPSK), the conventional distributed QO-STBC scheme (QPSK) and the distributed O-STBC network scheme (16QAM) for the transmission of $1 \mathrm{bits} / \mathrm{s} / \mathrm{Hz}$. And in Figure $2 b$, we show the performance of three schemes for the transmission of $2 \mathrm{bits} / \mathrm{s} / \mathrm{Hz}$.

From the simulation results, in Figure 2a, we can see that the proposed distributed STBC network scheme can achieve the full diversity, and the coding gain also increased. Simulation results show that the conventional distributed QO-STBC network scheme has better performance than the distributed O-STBC network scheme for the $1 \mathrm{bits} / \mathrm{s} / \mathrm{Hz}$ transmission at low SNR, and it starts to deteriorate due to diversity loss when the SNR value exceeds $22 \mathrm{~dB}$. The proposed network scheme outperform the layered Alamouti network scheme and the conventional QO-STBC network scheme about 4 and $5 \mathrm{~dB}$ at $10^{-3}$ BER due to the increase of diversity gain and coding gain. The performance of the proposed distributed STBC network scheme outperform the conventional QOSTBC network scheme about $7 \mathrm{~dB}$ at $10^{-3}$ BER due to the increase of rate. Also, we select three model, the conventional distributed QO-STBC scheme (16QAM), the proposed distributed STBC network scheme (16QAM) and the layered Alamouti network scheme (QPSK), and do the simulations for the transmission of $2 \mathrm{bits} / \mathrm{s} / \mathrm{Hz}$ in Figure $2 \mathrm{~b}$. It is clear that the proposed network scheme has the best performance. The analysis fits well in with simulation results.

\section{Conclusions}

In this article, we propose a simple distributed STBC network scheme with two relay nodes, in which each relay node is equipped with two receiving antennas and one transmission antenna. The successive OFDM symbols are transmitted from the source node to the destination node via two relay nodes over frequency selective fading channels. In other word, in the first step, the source node broadcast four OFDM symbols to the relay nodes. Then, at the relay nodes, the received signals are implemented 


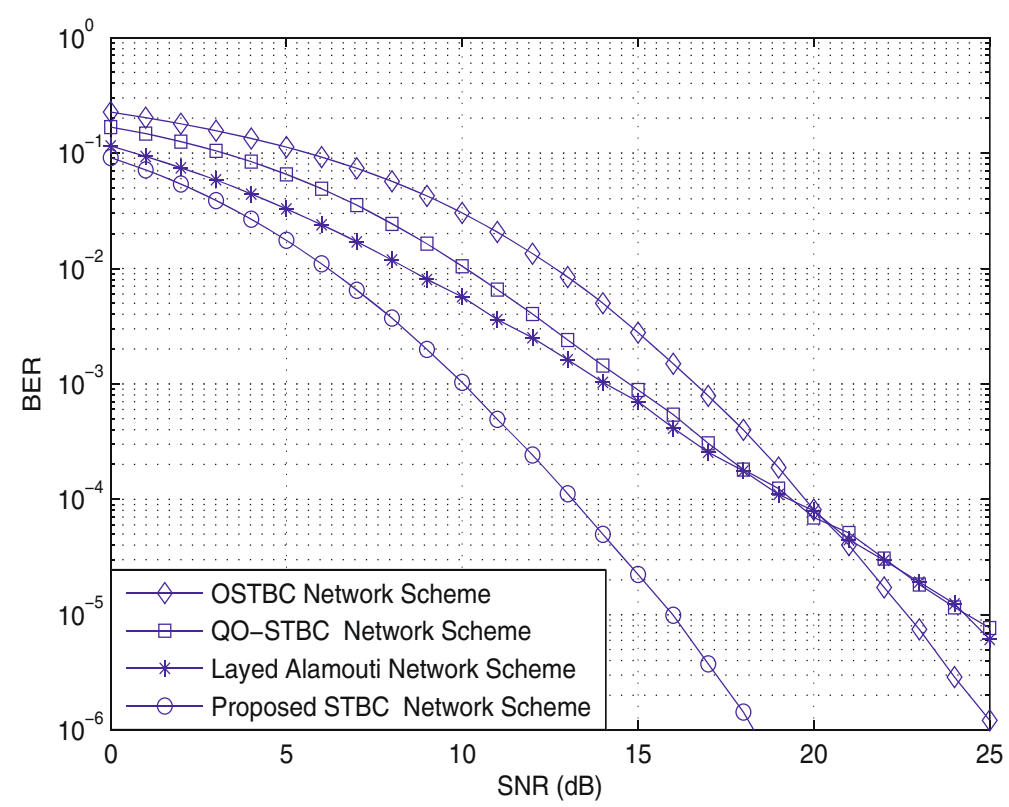

(a) Transmission rate $1 \mathrm{bits} / \mathrm{s} / \mathrm{Hz}$

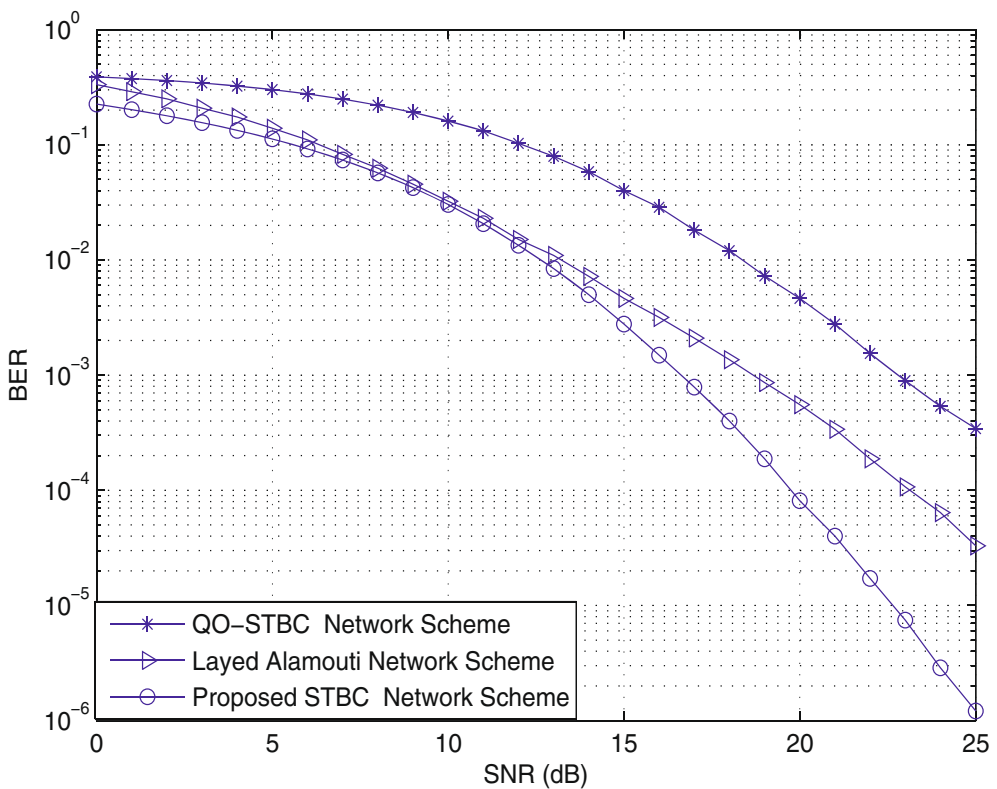

(b) Transmission rate $2 \mathrm{bits} / \mathrm{s} / \mathrm{Hz}$

Figure 2 Performance of the proposed DSTBC network scheme with frequency selective rayleigh flat fading channel. (a) Transmission rate 1 bits $/ \mathrm{s} / \mathrm{Hz}$. (b) Transmission rate 2 bits $/ \mathrm{s} / \mathrm{Hz}$.

with a simply operation and forwarded to the destination node.

During the whole processing, the equivalent QO-STBC with the full rate is implementing, and we received an equivalent half diversity orthogonal transmission code at the receiver through signals separated and re-constructed.
Thus, the proposed distributed network scheme achieve the full rate and full diversity at the process of transmission and receiver, respectively. So, the fast linear decoding algorithm can be used at the detector, and the decoding complexity is reduced obviously. Simulation results show that the performance of the proposed scheme has 
remarkable improvement due to the increase of coding gain and diversity gain. Also, it has new approach for the design of the future distributed STBC network scheme.

\section{Competing interests}

The authors declare that they have no competing interests.

\section{Acknowledgements}

This work was supported by the World Class University (WCU) R32-2012-00020014-0, BSRP2010-0020942, MEST 2012-002521, NRF, Korea, and the Natural Science Foundation of Liaoning Province (No. 201102076), China.

\section{Author details}

${ }^{1}$ College of Information Technology, Eastern liaoning university, Dandong, 118003 , P. R. China. ${ }^{2}$ College of Foreign Language, Eastern liaoning university, Dandong, 118003, P. R. China. ${ }^{3}$ Institute of Information and Communication, Chonbuk National University, 664-14 Deokjin-dong, Jeonju 561-756, Republic of Korea.

Received: 4 January 2012 Accepted: 17 July 2012

Published: 17 July 2012

\section{References}

1. A Alamouti, A simple transmit diversity scheme for wireless communications. IEEE J. Sel. Areas Commun. 16, 1451-1458 (1998)

2. VTarokh, H Jafarkhani, AR Calderbank, Space-time block codes from orthogonal designs. IEEE Trans. Inf. Theory. 45, 1456-1467 (1999)

3. H Jafarkhani, A quasiorthogonal space time block encodes. IEEE Trans. Commun. 49, 1-4 (2001)

4. O Tirkkonen, A Boariu, A Hottinen, Minimal nonorthogonality rate 1 space-time block code for 3+ Tx antennas. in Proc. IEEE 6th Int. Symp Spread-Spectrum Techniques and Applications (ISSSTA 2000), Volume 2. (Parsippany, New Jersey, USA, 429-432, 2000)

5. XW Su, Xia, Signal constellations for quasi-orthogonal space-time block codes with full diversit. IEEE Trans. Inf. Theory. 50, 2331-2347 (2004)

6. JN Laneman, DNC Tse, GW Wornell, Cooperative diversity in wireless networks: Efficient protocols and outage behavior. IEEE Trans. Inf. Theory (in press). 50, 3062-3080

7. JN Laneman, GW Wornell, Distributed Space-Time Coded Protocols for Exploiting Cooperative Diversity in Wireless Networks. IEEE Trans. Inf Theory. 59(10), 2415-2525 (2003)

8. A Sendonaris, E Erkip, B Aazhang, User cooperation diversity. Part I: System description. IEEE Trans. Commun. 51, 1927-1938 (2003)

9. A Sendonaris, E Erkip, B Aazhang, User cooperation diversity. Part II: implementation aspects and performance analysis. IEEE Trans. Commun. 51, 1939-1948 (2003)

10. Y Jing, B Hassibi, Distributed space-time coding in wireless relay networks. IEEE Trans. Wirel. Commun. 5(12), 3524-3536 (2006)

11. Z Li, X-G Xia, B Li, Achieving full diversity and fast ML decoding via simple analog network coding for asynchronous two-way relay networks. IEEE Trans. Commun. 57(12), 3672-3681 (2009)

12. JG Proakis, DG Manolakis, Digital Signal Processing: Principles, Algorithms and Applications. 3rd edn. (New Delhi: Prentice-Hall, 1996)

\section{Submit your manuscript to a SpringerOpen ${ }^{\odot}$ journal and benefit from:}

- Convenient online submission

- Rigorous peer review

- Immediate publication on acceptance

- Open access: articles freely available online

- High visibility within the field

- Retaining the copyright to your article 